\title{
One-dimensional nonlinear chromatography system and delta-shock waves
}

\author{
Guodong Wang
}

\begin{abstract}
The Riemann problem for the nonlinear chromatography system is considered. Existence and admissibility of $\delta$-shock type solution in both variables are established for this system. By the interactions of $\delta$-shock wave with elementary waves, the generalized Riemann problem for this system is presented, the global solutions are constructed, and the large time-asymptotic behavior of the solutions are analyzed. Moreover, by studying the limits of the solutions as perturbed parameter $\varepsilon$ tends to zero, one can observe that the Riemann solutions are stable for such perturbations of the initial data.
\end{abstract}

Mathematics Subject Classification. 35L65 - 35L67 · 35J70.

Keywords. Nonlinear chromatography system $\cdot$ Riemann problem $\cdot$ Generalized Riemann problem $\cdot \delta$-Shock wave.

\section{Introduction}

In this paper, we are concerned with the following conservation laws:

$$
\left\{\begin{array}{l}
\partial_{t} u+\partial_{x}\left(\frac{u}{1-u+v}\right)=0, \\
\partial_{t} v+\partial_{x}\left(\frac{v}{1-u+v}\right)=0
\end{array}\right.
$$

where $u, v$ are the nonnegative functions of the variables $(x, t) \in R \times R_{+}$, which express the concentrations of the two adsorbing species and $u \geq 0, v \geq 0,1-u+v>0$. Our main purpose is to investigate the existence and admissibility of nonclassical solutions and the generalized Riemann problem for the nonlinear chromatography system. The motivation comes from the fact that the delta-shock wave was captured numerically and experimentally by Mazzotti et al. [22-24] in the Riemann solutions for the local equilibrium model of two-component nonlinear chromatography, which consists of the following conservation laws

$$
\left\{\begin{array}{l}
\partial_{t}\left(u+\frac{a_{1} u}{1-u+v}\right)+\partial_{x} u=0, \\
\partial_{t}\left(u+\frac{a_{2} v}{1-u+v}\right)+\partial_{x} v=0,
\end{array}\right.
$$

where $u$ and $v$ are the concentrations of the adsorbing species, and $u \geq 0, v \geq 0,1-u+v>0, a_{2}>a_{1}>0$.

There is substantial difference between the nonlinear chromatography system (1.1) and the following chromatography equations

Research partially supported by the National Science Foundation of China (10971130), the Science Key Project of Education Department of Anhui Province (KJ2012A055) and the Doctoral Research Fund of Anhui University of Architecture (2011101-6). 


$$
\left\{\begin{array}{l}
\partial_{t} u+\partial_{x}\left(\frac{u}{1+u+v}\right)=0, \\
\partial_{t} v+\partial_{x}\left(\frac{v}{1+u+v}\right)=0,
\end{array}\right.
$$

where $u, v$ are nonnegative functions of the variables $(x, t) \in R \times R_{+}$. That is, the fact that $1-u+v>0$ in (1.1) and $1+u+v>1$ in (1.3), which results in the essential difference of the Riemann solutions, i.e., the former contains delta-shock solutions. For the Riemann solutions to the system (1.1), we can refer to [9] and the references cited therein. The system (1.1) belongs to so-called Temple class [39] and the shock curves coincide with the rarefaction curves in the phase plane. On the basis of the fact, we call the system (1.1) a generalized Temple class and the results about the Temple class are available for the system (1.1). Thanks to its features, well-posedness results for Temple systems are available for a much larger class of initial data compared to general systems of conservation laws. For the related results about Temple systems, we can refer to $[1-4,6]$ and the references cited therein.

Delta-shock wave is a kind of nonclassical nonlinear waves on which at least one of the state variables becomes a singular measure. Korchinski [18] introduced the concept of the Dirac function into the classical weak solution when he studied the Riemann problem for the following system

$$
\left\{\begin{array}{l}
u_{t}+\left(\frac{1}{2} u^{2}\right)_{x}=0, \\
v_{t}+\left(\frac{1}{2} u v\right)_{x}=0,
\end{array}\right.
$$

in his unpublished Ph.D. thesis in 1977. In fact, the concept of the $\delta$-shock solution and the corresponding Rankine-Hugoniot condition were also presented by Zeldovich and Myshkis [43] in the case of the continuity equation. Tan et al. [38] considered the system

$$
\left\{\begin{array}{l}
u_{t}+\left(u^{2}\right)_{x}=0, \\
v_{t}+(u v)_{x}=0,
\end{array}\right.
$$

and discovered that the form of Dirac delta functions supported on shocks was used as parts in their Riemann solutions for certain initial data. There is another well-known example, i.e. the transport equations

$$
\left\{\begin{array}{l}
\rho_{t}+(\rho u)_{x}=0, \\
(\rho u)_{t}+\left(\rho u^{2}\right)_{x}=0,
\end{array}\right.
$$

which are called the one-dimensional system of pressureless Euler equations. The transport equations (1.6) have been analyzed extensively, see $[5,8,14,15,19-21,35,41]$ and so on. Recently, the weak asymptotics method was widely used to study the $\delta$-shock wave type solution by Danilov et al. $[12,13,29,32,40]$ in the case of systems which are linear with respect to one of unknown functions. In the same papers, it is introduced a concept allowing functions of the form

$$
u(x, t)=u^{0}(x, t)+e(t) \delta(x-x(t)),
$$

to represent a solution to the considered systems. This concept is extended in [16] on systems which are nonlinear with respect to both variables. we also see papers $[17,25,27,34,42,43]$ for the related equations and results.

The paper is organized as follows. In Sect. 2, following [9], we consider the elementary waves to the system (1.1). In Sect. 2, the definition of $\delta$-shock wave type solution is given. Furthermore, under the generalized Rankine-Hugoniot condition and $\delta$-entropy condition, the $\delta$-shock wave solutions are obtained. In Sect. 4, we consider the initial value problem with three constant states. With the help of the interactions of the $\delta$-shock and elementary waves, the global solutions are constructed. Moreover, we prove that the solutions of the perturbed initial value problem converge to the corresponding Riemann solutions as $\varepsilon \rightarrow 0$, which shows the stability of the Riemann solutions for the small perturbation, and analyze the large time-asymptotic behavior of the solutions. 


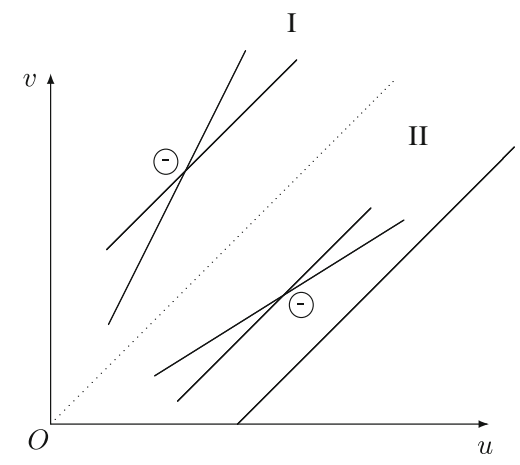

FIG. 1. Curves of elementary waves

\section{Elementary waves}

In this section, we will consider the Riemann problem of system (1.1) with the following Riemann initial data

$$
(u, v)(x, 0)=\left(u_{ \pm}, v_{ \pm}\right), \quad \pm x>0
$$

where $u_{ \pm}, v_{ \pm}$are constants and satisfy

$$
u_{ \pm} \geq 0, \quad v_{ \pm} \geq 0, \quad 1-u_{ \pm}+v_{ \pm}>0 .
$$

For details about the corresponding Riemann solutions, we can refer the reader to [9] and references cited therein, also see [37].

The eigenvalues of system (1.1) are

$$
\lambda_{1}=\frac{1}{1-u+v}, \quad \lambda_{2}=\frac{1}{(1-u+v)^{2}},
$$

and the corresponding right eigenvectors are

$$
\overrightarrow{r_{1}}=(1,1)^{T}, \quad \overrightarrow{r_{2}}=(u, v)^{T}
$$

By simple calculations, we have

$$
\nabla \lambda_{1} \cdot \overrightarrow{r_{1}}=0, \quad \nabla \lambda_{2} \cdot \overrightarrow{r_{2}} \neq 0,
$$

where $\nabla$ denotes the gradient with respect to $(u, v)$. Hence, $\lambda_{1}$ is linearly degenerate, $\lambda_{2}$ is genuinely nonlinear, and system (1.1) is nonstrictly hyperbolic for $1-u+v=1$. It is easy to obtain that the Riemann invariants of system (1.1) are

$$
w=v-u, \quad z=v / u .
$$

If $1-u+v=1$, one can obtain $\lambda_{1}=\lambda_{2}=1$, and we divide the phase plane into two parts (see Fig. 1)

$$
\mathrm{I}:\{(u, v) \mid v>u, u \geq 0\} \quad \text { and } \quad \mathrm{II}:\{(u, v) \mid u-1<v<u, v \geq 0\} .
$$

Riemann problems allow to consider the so-called self-similar solution, that is, the solution depending only on the self-similar variable $\xi=\frac{x}{t}$. By using self-similar transformation, system (1.1) becomes

$$
\left\{\begin{array}{l}
-\xi u_{\xi}+\left(\frac{u}{1-u+v}\right)_{\xi}=0 \\
-\xi v_{\xi}+\left(\frac{v}{1-u+v}\right)_{\xi}=0
\end{array}\right.
$$


and the Riemann initial data (2.1) can be changed into the following infinity boundary value

$$
(u, v)( \pm \infty)=\left(u_{ \pm}, v_{ \pm}\right) .
$$

For smooth solutions, system $(2.7)$ can be rewritten into

$$
A(U) U_{\xi}=0
$$

where $U=(u, v)^{T}$ and

$$
A(u, v)=\left(\begin{array}{cc}
-\xi+\frac{1+v}{(1-u+v)^{2}} & \frac{-u}{(1-u+v)^{2}} \\
\frac{v}{(1-u+v)^{2}} & -\xi+\frac{1-u}{(1-u+v)^{2}}
\end{array}\right)
$$

For a fixed left state $\left(u_{-}, v_{-}\right)$, the possible states which can be connected to $\left(u_{-}, v_{-}\right)$on the right by a rarefaction wave lie on a curve, which is given as follows

$$
R\left(u_{-}, v_{-}\right):\left\{\begin{array}{l}
\xi=\frac{1}{(1-u+v)^{2}}, \\
\frac{v}{u}=\frac{v_{-}}{u_{-}}, \\
v<v_{-}, u<u_{-}, \quad \text { if }\left(u_{-}, v_{-}\right) \in \mathrm{I}, \quad \text { or } \quad v>v_{-}, u>u_{-}, \quad \text { if }\left(u_{-}, v_{-}\right) \in \mathrm{II} .
\end{array}\right.
$$

In is well known that the Rankine-Hugoniot condition for a bounded discontinuity takes the form

$$
\left\{\begin{array}{l}
\sigma[u]=\left[\frac{u}{1-u+v}\right] \\
\sigma[v]=\left[\frac{v}{1-u+v}\right]
\end{array}\right.
$$

where [.] denotes the jump across the discontinuity and $\xi=\sigma$ denotes the speed of the discontinuity.

Let $P=1-u+v$, we have

$$
\left\{\begin{aligned}
\sigma & =\frac{1}{P_{r} P_{\ell}}+\frac{u_{r} v_{\ell}-u_{\ell} v_{r}}{P_{r} P_{\ell}\left(u_{r}-u_{\ell}\right)} \\
\sigma & =\frac{1}{P_{r} P_{\ell}}+\frac{u_{r} v_{\ell}-u_{\ell} v_{r}}{P_{r} P_{\ell}\left(v_{r}-v_{\ell}\right)}
\end{aligned}\right.
$$

where $P_{\ell}=1-u_{\ell}+v_{\ell}$ and $P_{r}=1-u_{r}+v_{r}$. From Eq. (2.13), we have

$$
\left(u_{r} v_{\ell}-u_{\ell} v_{r}\right)\left[\left(u_{r}-u_{\ell}\right)-\left(v_{r}-v_{\ell}\right)\right]=0 .
$$

Thus, we have $\left(u_{r} v_{\ell}-u_{\ell} v_{r}\right)=0$ or $u_{r}-v_{r}=u_{\ell}-v_{\ell}$. The former gives

$$
\frac{v_{r}}{u_{r}}=\frac{v_{\ell}}{u_{\ell}} \text {. }
$$

We also obtain

$$
\sigma=\frac{1}{P_{\ell} P_{r}} .
$$

For a shock wave, the Lax entropy conditions imply $1 / P_{r}<1 / P_{\ell}$ which means $P_{r}>P_{\ell}$. Therefore, for a fixed left state $\left(u_{-}, v_{-}\right)$, the possible states which can be connected to $\left(u_{-}, v_{-}\right)$on the right by a shock wave satisfy

$$
S\left(u_{-}, v_{-}\right):\left\{\begin{array}{l}
\sigma=\frac{1}{P P_{-}}=\frac{1}{(1-u+v)\left(1-u_{-}+v_{-}\right)} \\
\frac{v}{u}=\frac{v_{-}}{u_{-}}, \\
u>u_{-}, v>v_{-} \quad \text { if }\left(u_{-}, v_{-}\right) \in \mathrm{I} \text { or } u<u_{-}, v<v_{-} \quad \text { if }\left(u_{-}, v_{-}\right) \in \mathrm{II} .
\end{array}\right.
$$


The equality $v_{r}-u_{r}=v_{\ell}-u_{\ell}$ implies $1 /\left(1-u_{r}+v_{r}\right)=1 /\left(1-u_{\ell}+v_{\ell}\right)$, which means a contact discontinuity. Then, the possible states that can be connected to $\left(u_{-}, v_{-}\right)$on the right by a contact discontinuity lie on the curve

$$
J: \quad \frac{1}{1-u_{-}+v_{-}}=\frac{1}{1-u+v} .
$$

Using these classical elementary waves, one can construct the solutions as follows:

(a) The solution is $R+J$ if $\left(u_{-}, v_{-}\right) \in \mathrm{I}$ and $-u_{+}+v_{+}<-u_{-}+v_{-}$;

(b) The solution is $S+J$ if $\left(u_{-}, v_{-}\right) \in \mathrm{I}$ and $-u_{+}+v_{+}>-u_{-}+v_{-}$;

(c) The solution is $R+(0,0)+R$ if $\left(u_{-}, v_{-}\right) \in \mathrm{I}$ and $\left(u_{+}, v_{+}\right) \in \mathrm{II}$;

(d) The solution is $J+R$ if $\left(u_{-}, v_{-}\right) \in \mathrm{II}$ and $-u_{+}+v_{+}<-u_{-}+v_{-}$;

(e) The solution is $J+S$ if $\left(u_{-}, v_{-}\right) \in \mathrm{II}$ and $-u_{+}+v_{+}>-u_{-}+v_{-}$;

(f) $\left(u_{-}, v_{-}\right) \in \mathrm{II},\left(u_{+}, v_{+}\right) \in \mathrm{I}\left(\right.$ or $\left.1-u_{+}+v_{+}=1\right)$ or $1-u_{-}+v_{-}=1,\left(u_{+}, v_{+}\right) \in \mathrm{I}$. In this case (f), one can see

$$
\begin{aligned}
\lambda_{2}^{+} & =\frac{1}{\left(1-u_{+}+v_{+}\right)^{2}} \leq \lambda_{1}^{+}=\frac{1}{1-u_{+}+v_{+}} \\
& <\lambda_{1}^{-}=\frac{1}{1-u_{-}+v_{-}} \leq \lambda_{2}^{-}=\frac{1}{\left(1-u_{-}+v_{-}\right)^{2}},
\end{aligned}
$$

which implies that the solution cannot be constructed by applying these classical waves described above. Hence, the Riemann solution containing a weighted $\delta$-measure supported on a line should be constructed in order to established the existence.

\section{The nonclassical solutions}

In this section, we will consider the nonclassical solutions for system (1.1). Following [12,13,16], we have the following definitions.

Suppose that $\Gamma=\left\{\gamma_{i}: i \in I\right\}$ is a graph in the closed upper half-plane $\{(x, t): x \in \mathbb{R}, t \in[0,+\infty)\} \subset$ $\mathbb{R}^{2}$ containing smooth $\operatorname{arc} \gamma_{i}=\left\{(x, t): S_{i}(x, t)=0\right\}, i \in I$, and $I$ is a finite set. Let $I_{0}$ be subset of $I$ such that an $\operatorname{arc} \gamma_{i}$ for $i \in I_{0}$ starts from the points of the $x$-axis; $\Gamma_{0}=\left\{x_{k}^{0} ; i \in I_{0}\right\}$ is the set of initial points of arc $\gamma_{i}, i \in I_{0}$.

Consider $\delta$-shock wave type initial data $\left(u^{0}(x), v^{0}(x)\right)$, where

$$
u^{0}(x)=u_{0}(x)+e_{u}^{0} \delta\left(\Gamma_{0}\right), \quad v^{0}(x)=v_{0}(x)+\omega_{v}^{0} \delta\left(\Gamma_{0}\right),
$$

$u_{0}, v_{0} \in L^{\infty}\left(\mathbb{R} \times \mathbb{R}_{+}\right), e_{u}^{0} \delta\left(\Gamma_{0}\right)=\sum_{i \in I} e_{i}^{0} \delta\left(x-x_{i}^{0}\right)$ and $\omega_{v}^{0} \delta\left(\Gamma_{0}\right)=\sum_{i \in I} \omega_{i}^{0} \delta\left(x-x_{i}^{0}\right), e_{i}^{0}$ and $\omega_{i}^{0}$ are constants for $i \in I_{0}$.

Definition 3.1. A pair of distributions $(u(x, t), v(x, t))$ and a graph $\Gamma$, where $(u(x, t), v(x, t))$ has the form of the sum

$$
u(x, t)=\widehat{u}(x, t)+e(x, t) \delta(\Gamma) \quad \text { and } \quad v(x, t)=\widehat{v}(x, t)+\omega(x, t) \delta(\Gamma),
$$

$\widehat{u}, \widehat{v} \in L^{\infty}\left(\mathbb{R} \times \mathbb{R}_{+}\right)$and $e(x, t) \delta(\Gamma)=\sum_{i \in I} e_{i}(x, t) \delta\left(\gamma_{i}\right), \omega(x, t) \delta(\Gamma)=\sum_{i \in I} \omega_{i}(x, t) \delta\left(\gamma_{i}\right), e_{i}(x, t), \omega_{i}(x, t) \in$ $C(\Gamma)$ for $i \in I$, is called a generalized $\delta$-shock wave type solution of system (1.1) with the $\delta$-shock wave type initial data $\left(u^{0}(x), v^{0}(x)\right)$ if the integral identities 


$$
\left\{\begin{array}{l}
\int_{\mathbb{R}_{+}} \int_{\mathbb{R}}\left(\widehat{u} \phi_{t}+\frac{\widehat{u}}{1-\widehat{u}+\widehat{v}} \phi_{x}\right) \mathrm{d} x \mathrm{~d} t+\sum_{i \in I} \int_{\gamma_{i}} e_{i}(x, t) \frac{\partial \phi(x, t)}{\partial \ell} \mathrm{d} \ell \\
+\int_{\mathbb{R}} u_{0}(x) \phi(x, 0) \mathrm{d} x+\sum_{i \in I_{0}} e_{i}^{0} \phi\left(x_{i}^{0}, 0\right)=0, \\
\int_{\mathbb{R}_{+}} \int_{\mathbb{R}}\left(\widehat{v} \phi_{t}+\frac{\widehat{v}}{1-\widehat{u}+\widehat{v}} \phi_{x}\right) \mathrm{d} x \mathrm{~d} t+\sum_{i \in I} \int_{\gamma_{i}} \omega_{i}(x, t) \frac{\partial \phi(x, t)}{\partial \ell} \mathrm{d} \ell \\
+\int_{\mathbb{R}} v_{0}(x) \phi(x, 0) \mathrm{d} x+\sum_{i \in I_{0}} \omega_{i}^{0} \phi\left(x_{i}^{0}, 0\right)=0,
\end{array}\right.
$$

hold for all test functions $\phi(x, t) \in \mathcal{D}\left(R \times R_{+}\right)$, where $\frac{\partial \phi(x, t)}{\partial \ell}$ is the tangential derivative on the graph $\Gamma, \int_{\gamma_{i}} \mathrm{~d} \ell$ is a line integral over the $\operatorname{arc} \gamma_{i}$.

Theorem 3.2. For the Cauchy problem (1.1) and (2.1), in case (f), system (1.1) has $\delta$-shock wave type solution

$$
\begin{aligned}
& u(x, t)=u_{-}+[u] H(x-x(t))+e(t) \delta(x-x(t)), \\
& v(x, t)=v_{-}+[v] H(x-x(t))+\omega(t) \delta(x-x(t)),
\end{aligned}
$$

which satisfies the integral identities (3.1) in the sense of Definition 3.1 , where $\Gamma=\{(x, t): x=x(t)=$ $\left.\sigma_{\delta} t, t \geq 0\right\}$,

$$
\int_{\Gamma} e(x, t) \frac{\partial \phi(x, t)}{\partial \ell}=\int_{0}^{\infty} e(x, t) \frac{\mathrm{d} \phi(x, t)}{\mathrm{d} t} \mathrm{~d} t, \quad \int_{\Gamma} \omega(x, t) \frac{\partial \phi(x, t)}{\partial \ell}=\int_{0}^{\infty} \omega(x, t) \frac{\mathrm{d} \phi(x, t)}{\mathrm{d} t} \mathrm{~d} t,
$$

and $H(x)$ is the Heaviside function

$$
H(x)= \begin{cases}0, & \text { for } \quad x<0 \\ 1, & \text { for } \quad x>0\end{cases}
$$

In addition

$$
\left\{\begin{array}{l}
e(t)=\omega(t)=\frac{u_{-} v_{+}-v_{-} u_{+}}{\left(1-u_{-}+v_{-}\right)\left(1-u_{+}+v_{+}\right)} t, \\
\sigma_{\delta}=\frac{1}{\left(1-u_{-}+v_{-}\right)\left(1-u_{+}+v_{+}\right)} .
\end{array}\right.
$$

Proof. We need to check that the constructed $\delta$-measure solution satisfies the Definition 3.1 in the sense of distributions, that is,

$$
\begin{aligned}
& \int_{\mathbb{R}_{+}} \int_{\mathbb{R}}\left(\widehat{u} \phi_{t}+\frac{\widehat{u}}{1-\widehat{u}+\widehat{v}} \phi_{x}\right) \mathrm{d} x \mathrm{~d} t+\int_{0}^{\infty} e(t) \frac{\mathrm{d} \phi(x(t), t)}{\mathrm{d} t} \mathrm{~d} t+\int_{\mathbb{R}} u_{0}(x) \phi(x, 0) \mathrm{d} x=0, \\
& \int_{\mathbb{R}_{+}} \int_{\mathbb{R}}\left(\widehat{v} \phi_{t}+\frac{\widehat{v}}{1-\widehat{u}+\widehat{v}} \phi_{x}\right) \mathrm{d} x \mathrm{~d} t+\int_{0}^{\infty} \omega(t) \frac{\mathrm{d} \phi(x(t), t)}{\mathrm{d} t} \mathrm{~d} t+\int_{\mathbb{R}} v_{0}(x) \phi(x, 0) \mathrm{d} x=0,
\end{aligned}
$$

for all the test functions $\phi(x, t) \in \mathcal{D}\left(\mathbb{R} \times \mathbb{R}_{+}\right)$, where $u_{0}(x)=u_{-}+[u] H(x), v_{0}(x)=v_{-}+[v] H(x), \widehat{u}(x, t)=$ $u_{-}+[u] H(x-x(t))$ and $\widehat{v}(x, t)=v_{-}+[v] H(x-x(t))$. 
Denote by $\mathcal{A}$ the left-hand side of (3.3), we have

$$
\begin{aligned}
\mathcal{A}= & \int_{0}^{\infty} \int_{-\infty}^{x(t)}\left(u_{-} \phi_{t}+\frac{u_{-}}{1-u_{-}+v_{-}} \phi_{x}\right) \mathrm{d} x \mathrm{~d} t+\int_{0}^{\infty} \int_{x(t)}^{+\infty}\left(u_{+} \phi_{t}+\frac{u_{+}}{1-u_{+}+v_{+}} \phi_{x}\right) \mathrm{d} x \mathrm{~d} t \\
& +\int_{0}^{\infty} e(t) \frac{\mathrm{d} \phi(x(t), t)}{\mathrm{d} t} \mathrm{~d} t+\int_{-\infty}^{0} u_{-} \phi(x, 0) \mathrm{d} x+\int_{0}^{\infty} u_{+} \phi(x, 0) \mathrm{d} x .
\end{aligned}
$$

Without loss of generality, we assume $\sigma_{\delta}>0$, then the first term on the right-hand side of (3.5) equals

$$
\begin{aligned}
& \int_{0}^{\infty} \int_{-\infty}^{0} u_{-} \phi_{t} \mathrm{~d} x \mathrm{~d} t+\int_{0}^{\infty} \int_{0}^{x(t)} u_{-} \phi_{t} \mathrm{~d} x \mathrm{~d} t+\int_{0}^{\infty} \int_{-\infty}^{x(t)} \frac{u_{-}}{1-u_{-}+v_{-}} \phi_{x} \mathrm{~d} x \mathrm{~d} t \\
& =-\int_{-\infty}^{0} u_{-} \phi(x, 0) \mathrm{d} x+\int_{0}^{\infty} \int_{0}^{x(t)} u_{-} \phi_{t} \mathrm{~d} x \mathrm{~d} t+\int_{0}^{\infty} \frac{u_{-}}{1-u_{-}+v_{-}} \phi(x(t), t) \mathrm{d} t \\
& =-\int_{-\infty}^{0} u_{-} \phi(x, 0) \mathrm{d} x+\int_{0}^{\infty} \mathrm{d} x \int_{t(x)}^{\infty} u_{-} \phi_{t} \mathrm{~d} t+\int_{0}^{\infty} \frac{u_{-}}{1-u_{-}+v_{-}} \frac{1}{\sigma_{\delta}} \phi(x, t(x)) \mathrm{d} x \\
& =-\int_{-\infty}^{0} u_{-} \phi(x, 0) \mathrm{d} x+\int_{0}^{\infty}\left(\frac{1}{\sigma_{\delta}\left(1-u_{-}+v_{-}\right)}-1\right) u_{-} \phi(x, t(x)) \mathrm{d} x .
\end{aligned}
$$

The second term on the right-hand side of (3.5) equals

$$
\begin{aligned}
& \int_{0}^{\infty} \mathrm{d} x \int_{0}^{t(x)} u_{+} \phi_{t} \mathrm{~d} t-\int_{0}^{\infty} \frac{u_{+}}{1-u_{+}+v_{+}} \phi(x(t), t) \mathrm{d} t \\
& \quad=-\int_{0}^{\infty} u_{+} \phi(x, 0) \mathrm{d} x+\int_{0}^{\infty}\left(1-\frac{1}{\sigma_{\delta}\left(1-u_{+}+v_{+}\right)}\right) u_{+} \phi(x, t(x)) \mathrm{d} x .
\end{aligned}
$$

The third term on the right-hand side of (3.5) equals

$$
\begin{aligned}
& \left.\left(\sigma_{\delta}[u]-\left[\frac{u}{1-u+v}\right]\right) t \phi(x(t), t)\right|_{0} ^{+\infty}-\left(\sigma_{\delta}[u]-\left[\frac{u}{1-u+v}\right]\right) \int_{0}^{+\infty} \phi(x(t), t) \mathrm{d} t \\
& \quad=-\left(\sigma_{\delta}[u]-\left[\frac{u}{1-u+v}\right]\right) \frac{1}{\sigma_{\delta}} \int_{0}^{\infty} \phi(x, t(x)) \mathrm{d} x .
\end{aligned}
$$

It is clear from $(3.5)-(3.8)$ that

$$
\mathcal{A}=0 \text {. }
$$

A similar argument gives (3.4). It is easy to obtain (3.2). So, we complete the proof.

Using Definition 3.1 and repeating the proof of Theorem 3.2 almost word-for-word, one can derive the generalized Rankine-Hugoniot condition for $\delta$-shock wave type solution of system (1.1). 
Theorem 3.3. Suppose that $\Omega \subset R \times R_{+}$is some region cut by a smooth curve $\Gamma=\{(x, t): x=x(t)\}$ into a left- and right-hand parts $\left.\Omega_{ \pm}=\{(x, t): \pm(x-x(t))>0\},(u(x, t)), v(x, t)\right)$, is a generalized $\delta$-shock wave type solution of system (1.1), functions $\widehat{u}(x, t), \widehat{v}(x, t)$ are smooth in $\Omega_{ \pm}$, and have one-side limits $\widehat{u}_{ \pm}, \widehat{v}_{ \pm}$on the curve $\Gamma$. Then, the generalized Rankine-Hugoniot condition for $\delta$-shock is

$$
\left\{\begin{array}{l}
\frac{\mathrm{d} x(t)}{\mathrm{d} t}=\sigma_{\delta}, \\
\frac{\mathrm{d} e(t)}{\mathrm{d} t}=\left.\left(\dot{x}(t)[u]-\left[\frac{u}{1-u+v}\right]\right)\right|_{x=x(t)}, \\
\frac{\mathrm{d} \omega(t)}{\mathrm{d} t}=\left.\left(\dot{x}(t)[v]-\left[\frac{v}{1-u+v}\right]\right)\right|_{x=x(t)},
\end{array}\right.
$$

where $e(t) \doteq e(x(t), t), \omega(t) \doteq \omega(x(t), t)$ and $(\dot{)})=\frac{\mathrm{d}}{\mathrm{d} t}(\cdot)$.

In addition to the generalized Rankine-Hugoniot conditions (3.9), to guarantee uniqueness, the discontinuity should satisfy

$$
\begin{aligned}
\lambda_{1}^{+} & =\frac{1}{\left(1-u_{+}+v_{+}\right)^{2}} \leq \lambda_{2}^{+}=\frac{1}{1-u_{+}+v_{+}} \leq \sigma_{\delta} \\
& \leq \lambda_{1}^{-}=\frac{1}{1-u_{-}+v_{-}} \leq \lambda_{2}^{-}=\frac{1}{\left(1-u_{-}+v_{-}\right)^{2}}
\end{aligned}
$$

where $u_{ \pm}$and $v_{ \pm}$are the respective left- and right-hand limit values of $u(x, t)$ and $v(x, t)$ on the discontinuity curve. Condition (3.10) is called as $\boldsymbol{\delta}$-entropy condition. It is overcompressive and means that all the characteristic lines on both sides of the discontinuity are not out-coming. A discontinuity satisfying (3.9), (3.10), and (3.2) will be called a $\delta$-shock wave to system (1.1).

So, we complete the construction of the Riemann solutions to system (1.1).

\section{Interactions of $\delta$-shock wave with elementary waves}

To start off, we consider the initial value problem with three pieces constant states

$$
(u, v)(x, 0)= \begin{cases}\left(u_{-}, v_{-}\right), & x<0, \\ \left(u_{m}, v_{m}\right), & 0<x<\varepsilon, \\ \left(u_{+}, v_{+}\right), & x>\varepsilon,\end{cases}
$$

where $\varepsilon>0$ is arbitrarily small. The data (4.1) is a perturbation of the Riemann initial data (2.1). Our interest is to investigate whether the Riemann solutions of (1.1) and (2.1) are the limits of the solutions of (1.1) and (4.1) as $\varepsilon \rightarrow 0$. In this section, we only consider the interactions of the $\delta$-shock and elementary waves. For the interactions of elementary waves, we refer the readers to the book of Smoller [36] and the monograph of Chang and Hsiao [7]. Also see [33] for the recent work about the interactions of elementary waves. For a comprehensive survey, we can see the books written by Dafermos [10] and Serre [31]. For interaction with $\delta$-shocks, we can see [28] and the references cited therein. The problem can be divided into eleven cases as follows:

$$
\begin{aligned}
& J+R \oplus \delta, \quad J+S \oplus \delta, \quad R+R \oplus \delta, \quad \delta \oplus \delta, \quad R+J \oplus \delta, \\
& S+J \oplus \delta, \quad \delta \oplus R+J, \quad \delta \oplus S+J, \quad \delta \oplus J+R, \quad \delta \oplus J+S, \quad \delta \oplus R+R .
\end{aligned}
$$

When the Riemann solution $\mathcal{R}\left(\odot\right.$, (m) is of type $S+J$, we have $\left(u_{-}, v_{-}\right),\left(u_{m}, v_{m}\right) \in \mathrm{I}$, which implies that the $\delta$-shock cannot appear between left state $\left(u_{m}, v_{m}\right)$ and right state $\left(u_{+}, v_{+}\right)$. Thus, $S+J \oplus \delta$ is impossible for this situation. Similarly, $\delta \oplus J+S$ is also impossible. We will discuss the first five cases in detail and the other four cases can be discussed in a similar way. 

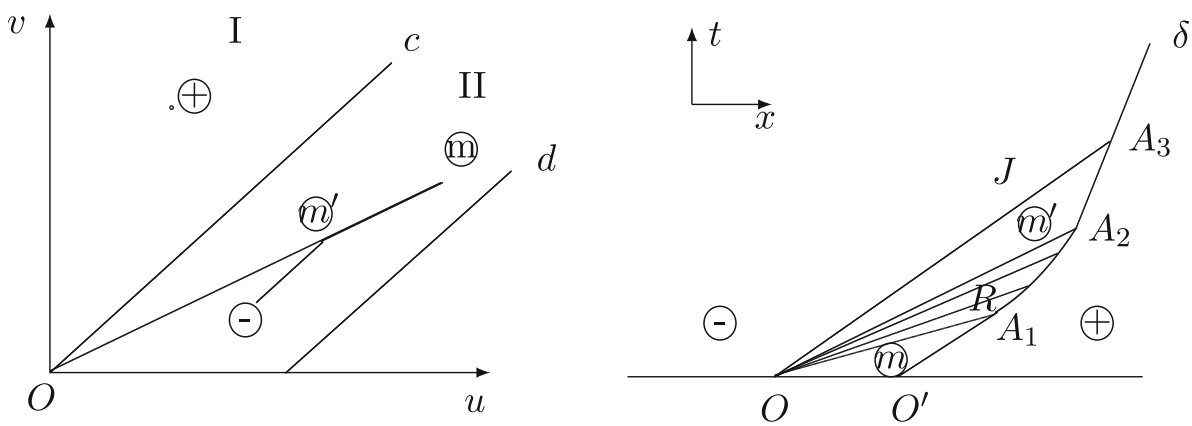

FIG. 2. Case 1.1: $\left(u_{-}, v_{-}\right) \in \mathrm{II},\left(u_{+}, v_{+}\right) \in \mathrm{I}$

For all the first three cases, we have $\left(u_{m}, v_{m}\right) \in$ II.

Case 1. $J+R \oplus \delta$-shock. According to the location of states $\left(u_{-}, v_{-}\right)$and $\left(u_{+}, v_{+}\right)$, there are three subcases: (1.1) $\left(u_{-}, v_{-}\right) \in \mathrm{II},\left(u_{+}, v_{+}\right) \in \mathrm{I} ;(1.2) 1-u_{-}+v_{-}=1,\left(u_{+}, v_{+}\right) \in \mathrm{I} ;(1.3) 1-u_{-}+v_{-}=1$ and $1-u_{+}+v_{+}=1$. In the following, we discuss them in detail.

Case 1.1. $\left(u_{-}, v_{-}\right) \in \mathrm{II},\left(u_{+}, v_{+}\right) \in \mathrm{I}$, see Fig. 2. A contact discontinuity and a rarefaction wave start at point $\mathrm{O}$. The speed of the $\delta$-shock is $\sigma_{\delta}=1 /\left(P_{m} P_{+}\right)<1 / P_{m}^{2}$, where $P_{m}=1-u_{m}+v_{m}$ and the rarefaction wave will overtake the $\delta$-shock at finite time. The intersection point $A_{1}\left(x_{1}, t_{1}\right)$ is determined by

$$
\left\{\begin{array}{l}
\frac{x}{t}=\frac{1}{P_{m}^{2}} \\
\frac{x-\varepsilon}{t}=\frac{1}{P_{m} P_{+}}
\end{array}\right.
$$

which implies

$$
\left(x_{1}, t_{1}\right)=\left(\frac{P_{+}}{P_{+}-P_{m}} \varepsilon, \frac{P_{+} P_{m}^{2}}{P_{+}-P_{m}} \varepsilon\right) .
$$

We have the following fact:

Lemma 4.1. The $\delta$-shock can penetrate the rarefaction wave $R$ and interacts with the contact discontinuity $J$, the interaction of the delta wave and the discontinuity yields a new $\delta$-shock.

Proof. When $\frac{1}{P_{m^{\prime}}} \leq \frac{1}{P} \leq \frac{1}{P_{m}}$, we have $\frac{1}{P_{+}}<1 \leq \frac{1}{P_{m^{\prime}}} \leq \frac{1}{P} \leq \frac{1}{P_{m}}$, then the $\delta$-shock entropy condition

$$
\frac{1}{P_{+}^{2}}<\frac{1}{P_{+}} \leq \sigma_{\delta}=\frac{1}{P P_{+}} \leq \frac{1}{P} \leq \frac{1}{P^{2}}
$$

is satisfied, where the intermediate state $\left(u_{m^{\prime}}, u_{m^{\prime}}\right)$ is expressed as

$$
\left\{\begin{array}{l}
\frac{1}{1-u_{m^{\prime}}+v_{m^{\prime}}}=\frac{1}{1-u_{-}+v_{-}}, \\
\frac{v_{m^{\prime}}}{u_{m^{\prime}}}=\frac{v_{m}}{u_{m}} .
\end{array}\right.
$$

The $\delta$-shock begins interacting with the rarefaction wave $R$ at the point $A_{1}$ and starts to bend. The bending of $\delta$-shock is determined by 


$$
\left\{\begin{array}{l}
\frac{x}{t}=\frac{1}{P^{2}}, \\
\frac{\mathrm{d} x}{\mathrm{~d} t}=\frac{1}{P P_{+}}, \quad \frac{1}{P_{m^{\prime}}} \leq \frac{1}{P} \leq \frac{1}{P_{m}} \\
\left.x\right|_{t=t_{1}}=\frac{P_{+}}{P_{+}-P_{m}} \varepsilon
\end{array}\right.
$$

Substituting the first equation of (4.5) into the second equation, we obtain

$$
\frac{\mathrm{d} x}{\mathrm{~d} t}=\frac{1}{P_{+}} \sqrt{\frac{x}{t}} .
$$

Differentiating Eq. (4.6) with respect to $t$, we get

$$
\frac{d^{2} x}{\mathrm{~d} t^{2}}=\frac{1}{2 P_{+} \sqrt{x t}}\left(\frac{\mathrm{d} x}{\mathrm{~d} t}-\frac{x}{t}\right) .
$$

Combining the first equation with the second equation in (4.5), it is easy to get

$$
\frac{d^{2} x}{\mathrm{~d} t^{2}}=\frac{1}{2 P P_{+} \sqrt{x t}}\left(\frac{1}{P_{+}}-\frac{1}{P}\right)<0
$$

for $\frac{1}{P_{+}}<\frac{1}{P}<\frac{1}{P_{m^{\prime}}}$. Equation (4.8) means that the $\delta$-shock decelerates during the process of penetration.

Integrating (4.6), we have

$$
\sqrt{x}=\sqrt{x_{1}}+\frac{1}{P_{+}}\left(\sqrt{t}-\sqrt{t_{1}}\right), \quad t>t_{1},
$$

which together with $x=\frac{1}{P_{m^{\prime}}^{2}} t$ determines the ending point $A_{2}\left(x_{2}, t_{2}\right)$ of the penetration. A direct calculation leads to

$$
\left\{\begin{aligned}
x_{2} & =\frac{P_{+}\left(P_{+}-P_{m}\right)}{\left(P_{+}-P_{m^{\prime}}\right)^{2}} \varepsilon, \\
t_{2} & =\frac{P_{+}\left(P_{+}-P_{m}\right) P_{m^{\prime}}^{2}}{\left(P_{+}-P_{m^{\prime}}\right)^{2}} \varepsilon .
\end{aligned}\right.
$$

After penetrating the $R$, the propagating speed of the new $\delta$-shock is $\frac{1}{P_{m^{\prime}} P_{+}}$, which is less than the speed of the contact discontinuity $\lambda=\frac{1}{P_{m^{\prime}}}=\frac{1}{P_{-}}$. So, the contact discontinuity $J$ will overtake the $\delta$-shock at a finite time. Their intersection point $A_{3}\left(x_{3}, t_{3}\right)$ is determined by

which means

$$
\left\{\begin{array}{l}
\frac{x}{t}=\frac{1}{P_{-}}=\frac{1}{P_{m^{\prime}}}, \\
\frac{\mathrm{d} x}{\mathrm{~d} t}=\frac{1}{P_{m^{\prime}} P_{+}}=\frac{1}{P_{-} P_{+}}, \\
\left.x\right|_{t=t_{2}}=x_{2},
\end{array}\right.
$$

$$
\left\{\begin{array}{l}
x_{3}=\left(\frac{P_{-}}{t_{2}}\right)^{\frac{1}{P_{+}-1}} x_{2}^{\frac{P_{+}}{P_{+}-1}}, \\
t_{3}=\left(P_{-} x_{2}\right)^{\frac{P_{+}}{P_{+}-1}} t_{2}^{\frac{1}{1-P_{+}}} .
\end{array}\right.
$$

After the time $t_{3}$, the new $\delta$-shock is determined by

$$
\left\{\begin{array}{l}
\frac{\mathrm{d} x}{\mathrm{~d} t}=\frac{1}{P_{-} P_{+}}, \\
\left.x\right|_{t=t_{3}}=x_{3},
\end{array}\right.
$$



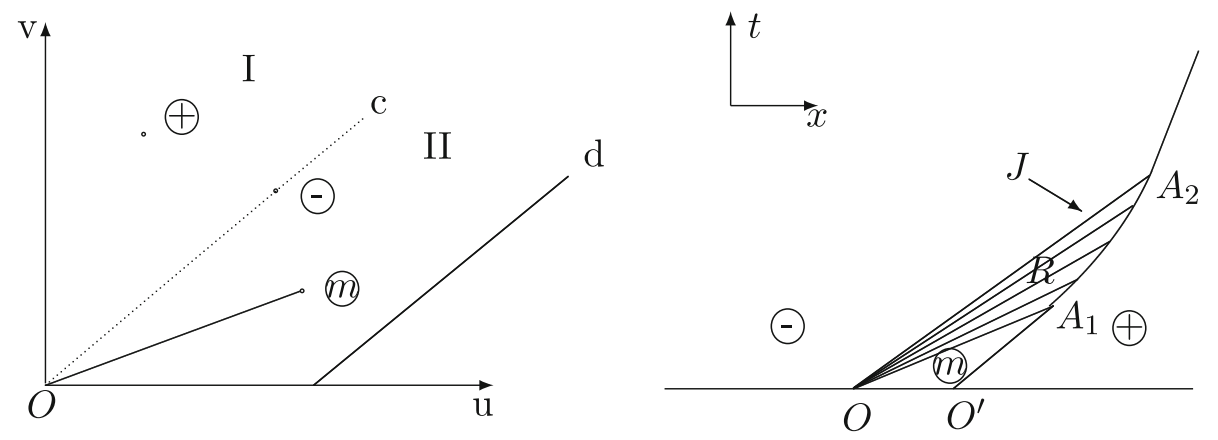

FIG. 3. Case 1.2: $1-u_{-}+v_{-}=1,\left(u_{+}, v_{+}\right) \in \mathrm{I}$

which implies

$$
x=\frac{1}{P_{-} P_{+}}\left(t-t_{3}\right)+x_{3} .
$$

From Fig. 2, we can see that as $t \rightarrow \infty$, the time-asymptotic solution can be described as

$$
\left(u_{-}, v_{-}\right)+\delta \text { shock }+\left(u_{+}, v_{+}\right)
$$

Moreover, letting $\varepsilon \rightarrow 0$, one can easily see that the limit of the solution (1.1) and (4.1) is the corresponding Riemann solution in this case.

We now seek the strength of the $\delta$-shock. Before the $\delta$-shock interacting with the $R$, the strength of the $\delta$-shock is determined by

$$
\left\{\begin{array}{l}
u_{\delta}=\frac{1}{P_{m_{m} P_{+}}}\left[\left(1-P_{m}\right) u_{+}+\left(P_{+}-1\right) u_{m}\right] t, \\
v_{\delta}=\frac{1}{P_{m} P_{+}}\left[\left(1-P_{m}\right) v_{+}+\left(P_{+}-1\right) v_{m}\right] t
\end{array}\right.
$$

where $t \leq t_{1}$. When $t_{1} \leq t \leq t_{2}$, the strength of the $\delta$-shock is determined by the following ordinary differential equations

$$
\left\{\begin{array}{l}
\frac{\mathrm{d} \omega_{u}}{\mathrm{~d} t}=\frac{1}{P P_{+}}\left[(1-P) u_{+}+\left(P_{+}-1\right) u\right], \quad P_{m} \leq P \leq P_{m^{\prime}} \\
\frac{\mathrm{d} \omega_{v}}{\mathrm{~d} t}=\frac{1}{P P_{+}}\left[(1-P) v_{+}+\left(P_{+}-1\right) v\right], \quad P_{m} \leq P \leq P_{m^{\prime}} \\
\left.\omega_{u}\right|_{t=t_{1}}=\frac{1}{P_{m} P_{+}}\left[\left(1-P_{m}\right) u_{+}+\left(P_{+}-1\right) u_{m}\right] t_{1}, \\
\left.\omega_{v}\right|_{t=t_{1}}=\frac{1}{P_{m} P_{+}}\left[\left(1-P_{m}\right) v_{+}+\left(P_{+}-1\right) v_{m}\right] t_{1},
\end{array}\right.
$$

where $P=\frac{\sqrt{t}}{\sqrt{x_{1}}+\left(\sqrt{t}-\sqrt{t_{1}}\right) / P_{+}}$, derived from Eqs. (4.5) and (4.9).

Case 1.2. $1-u_{-}+v_{-}=1,\left(u_{+}, v_{+}\right) \in \mathrm{I}$, see Fig. 3 .

The $\delta$-shock interacts with the rarefaction wave $R$ and the contact discontinuity $J$. The picture is shown in Fig. 3. Different from the above subcase 1.1, the $\delta$-shock penetrates the $R$ at point $A_{2}$ and meanwhile interacts with the $J$, then generates another new $\delta$-shock, see subcase 1.1 for detail.

As $t \rightarrow \infty$, the time-asymptotic solution can be described as

$$
\left(u_{-}, v_{-}\right)+\delta \text {-shock }+\left(u_{+}, v_{+}\right) .
$$

We can see that the limit of the solution (1.1) and (4.1) is the corresponding Riemann solution in this case. 

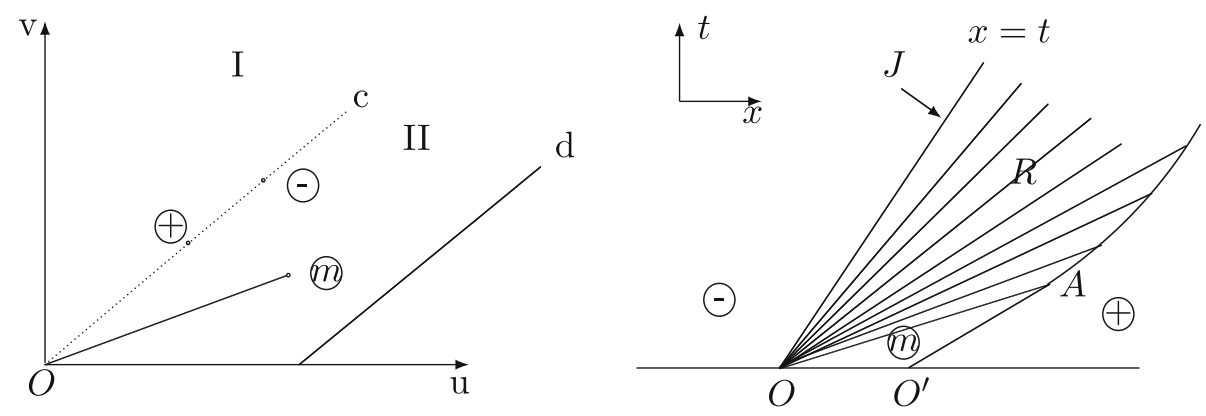

FIG. 4. Case 1.3: $1-u_{-}+v_{-}=1$, and $1-\left(u_{+}+v_{+}\right)=1$

Case 1.3. $1-u_{-}+v_{-}=1$ and $1-u_{+}+v_{+}=1$.

We have the following properties.

Lemma 4.2. The $\delta$-shock interacts with the rarefaction wave $R$, but cannot penetrate the $R$. Moreover, it has $x=t$ as its asymptote, see Fig. 4.

Proof. The $\delta$-shock begins to interact with the rarefaction wave $R$ at point $A\left(x_{1}, t_{1}\right)$ and changes into another new $\delta$-shock (a curve) which is calculated by

$$
\left\{\begin{array}{l}
\frac{x}{t}=\frac{1}{P^{2}}, \\
\frac{\mathrm{d} x}{\mathrm{~d} t}=\frac{1}{P P_{+}}, \quad 1=\frac{1}{P_{+}}=\frac{1}{P_{-}} \leq \frac{1}{P} \leq \frac{1}{P_{m}}, \\
\left.x\right|_{t=t_{1}}=x_{1} .
\end{array}\right.
$$

From (4.9), we have

$$
\sqrt{x}=\sqrt{x_{1}}+\frac{1}{P_{+}}\left(\sqrt{t}-\sqrt{t_{1}}\right) .
$$

The first equation in (4.19) implies $\sqrt{x}=\sqrt{t} / P$. Substituting it into (4.20), we get $\sqrt{t}=\left(\sqrt{x_{1}}-\right.$ $\left.\sqrt{t_{1}} / P_{+}\right) /\left(\frac{1}{P}-\frac{1}{P_{+}}\right)$which implies $t \rightarrow+\infty$, as $P \rightarrow P_{+}=P_{-}$. The fact tells us that the new $\delta$-shock cannot penetrate the $R$ and has $x=t / P_{-}^{2}=t$ as its asymptote.

As $t \rightarrow \infty$, the speed of the $\delta$-shock tends to $\sigma_{\delta}=1 /\left(P_{-} P_{+}\right)=1 / P_{-}=1 / P_{+}=1$ and the $\delta$-shock decelerates during the process of penetration. For large time, the solution can be expressed as

$$
\left(u_{-}, v_{-}\right)+J+\left(u_{+}, v_{+}\right)
$$

So, as $\varepsilon \rightarrow 0$, we can see that the limit of the solution (1.1) and (4.1) is the corresponding Riemann solution in this case.

Case 2. $J+S \oplus \delta$-shock, see Fig. 5 . In this case, we can describe the interactions by the following lemma.

Lemma 4.3. The shock overtakes the $\delta$-shock at a point $A_{1}\left(x_{1}, t_{1}\right)$ and generates a new $\delta$-shock, which is overtook by the contact discontinuity $J$ at finite time as well.

Proof. The propagation speed of the shock $S$ is $\sigma_{1}=\frac{1}{P_{m^{\prime}} P_{m}}$, which is greater than that of the $\delta$-shock, $\sigma_{2}=\frac{1}{P_{m} P_{+}}$. Then, the $S$ intersects with the $\delta$-shock at a point $A_{1}\left(x_{1}, t_{1}\right)$ which is calculated by

$$
\left\{\begin{array}{l}
x=\frac{1}{P_{m^{\prime}} P_{m}} t, \\
x=\frac{1}{P_{m} P_{+}} t+\varepsilon .
\end{array}\right.
$$



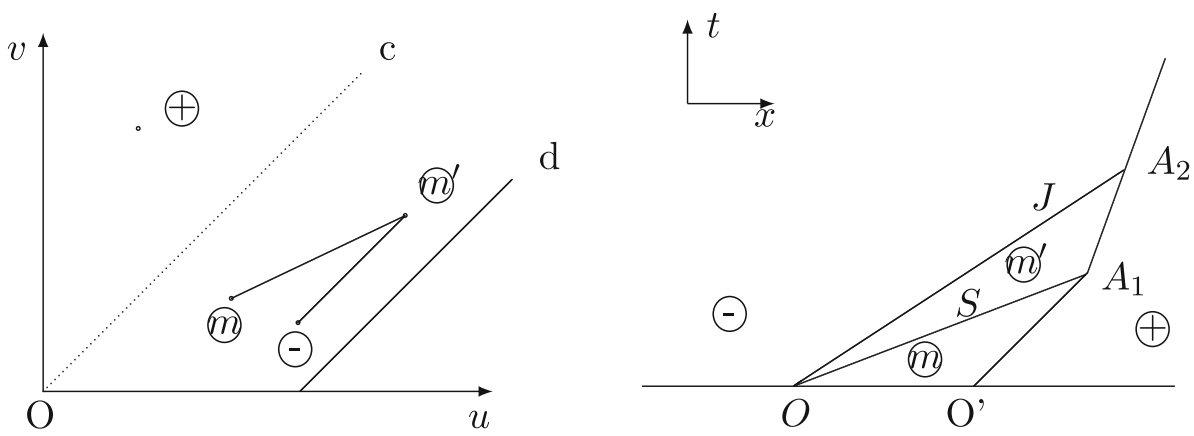

FIG. 5. Case 2: $J+S \oplus \delta$-shock

Using $P_{-}=P_{m^{\prime}}$, we have

$$
\left(x_{1}, t_{1}\right)=\left(\frac{P_{+}}{P_{+}-P_{-}} \varepsilon, \frac{P_{-} P_{m} P_{+}}{P_{+}-P_{-}} \varepsilon\right) .
$$

After the time $t_{1}$, the $J$ will overtake the new $\delta$-shock at point $A_{2}\left(x_{2}, t_{2}\right)$, which is determined by

$$
\left\{\begin{array}{l}
\frac{x}{t}=\frac{1}{P_{-}}=\frac{1}{P_{m^{\prime}}}, \\
\frac{\mathrm{d} x}{\mathrm{~d} t}=\frac{1}{P_{m^{\prime}} P_{+}}, \\
\left.x\right|_{t=t_{1}}=x_{1},
\end{array}\right.
$$

which implies

$$
\left.\left(x_{2}, t_{2}\right)=\left(\left(\frac{P_{-}}{t_{1}}\right)^{\frac{1}{P_{+}-1}} x_{1}^{\frac{P_{+}}{P_{+}-1}},\left(P_{-} x_{1}\right)^{\frac{P_{+}}{P_{+}^{-1}}} t_{1}^{\frac{1}{1-P_{+}}}\right)\right) .
$$

We know that when the new $\delta$-shock interacts with the $J$, it keeps the same speed, i.e., $\sigma=\frac{1}{P_{-} P_{+}}=\frac{1}{P_{m^{\prime}} P_{+}}$. Moreover, after the time $t_{2}$, the new $\delta$-shock is calculated by

$$
\left\{\begin{array}{l}
\frac{\mathrm{d} x}{\mathrm{~d} t}=\frac{1}{P_{-} P_{+}} \\
\left.x\right|_{t=t_{2}}=x_{2} .
\end{array}\right.
$$

Thus, as $t \rightarrow \infty$, the solution can be described as

$$
\left(u_{-}, v_{-}\right)+\delta \text {-shock }+\left(u_{+}, v_{+}\right) .
$$

Case 3. $R_{1}+R_{2} \oplus \delta$-shock. By Riemann solutions $\mathcal{R}(\Theta, \oplus)$ between state $\left(u_{-}, v_{-}\right)$and $\left(u_{+}, v_{+}\right)$, we divide this case into two subcases: $(3.1) \mathcal{R}(\Theta, \oplus)$ is $R+J$ and $(3.2) \mathcal{R}(\Theta, \oplus)$ is $S+J$.

Case 3.1. $\mathcal{R}(\odot, \oplus)$ is $R+J$. There are two kinds: (i), $\left(u_{-}, v_{-}\right),\left(u_{+}, v_{+}\right) \in \mathrm{I}$ and (ii) $\left(u_{-}, v_{-}\right) \in \mathrm{I}, 1-$ $u_{+}+v_{+}=1$.

(i). $\left(u_{-}, v_{-}\right),\left(u_{+}, v_{+}\right) \in \mathrm{I}$, see Fig. 6. By the above discussion, it is clear that the $\delta$-shock cannot penetrate the rarefaction wave $R_{2}$ at point $A_{2}\left(x_{2}, t_{2}\right)$, since when $\frac{1}{P_{-}} \leq \frac{1}{P}<1$, the $\delta$-shock entropy condition is not satisfied and its strength is equal to zero. At the time $t=t_{2}$, we again have a new Riemann problem with data $\left(u_{\ell}, v_{\ell}\right)=(0,0)$ and $\left(u_{r}, v_{r}\right)=\left(u_{+}, v_{+}\right)$, which is resolved by a shock $S$ and 

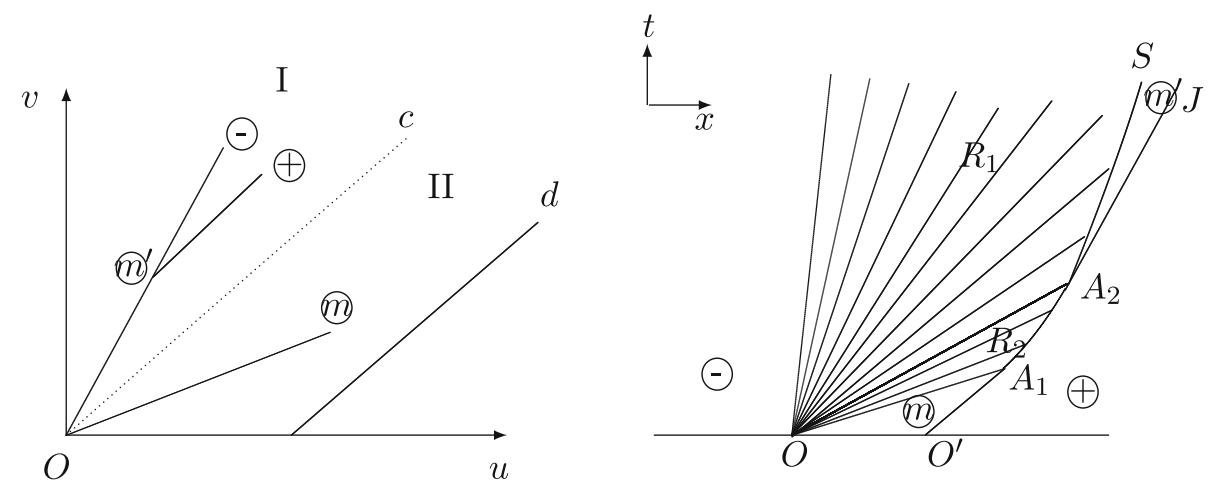

FIG. 6. Case 3.1: $\mathcal{R}(\odot, \oplus)$ is $R+J,(i) .\left(u_{-}, v_{-}\right),\left(u_{+}, v_{+}\right) \in \mathrm{I}$
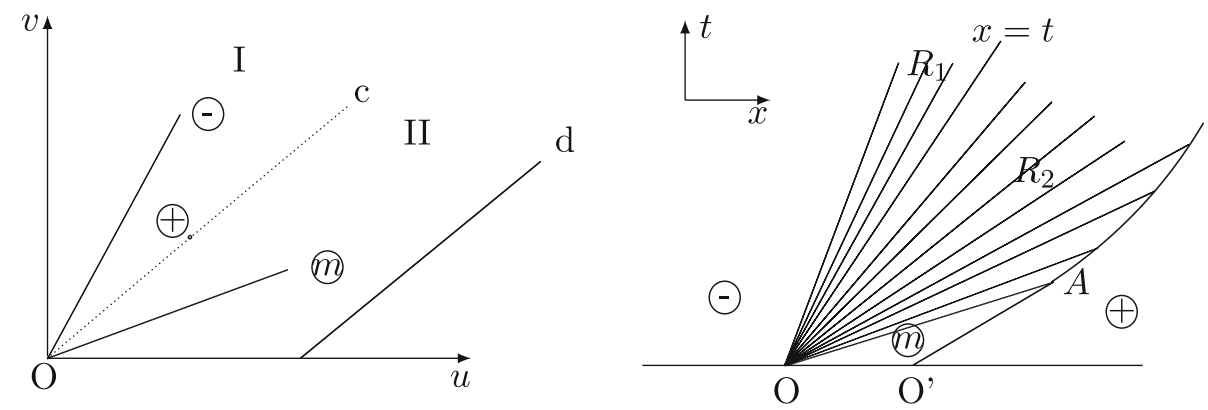

FIG. 7. Case 3.1: $\mathcal{R}(\odot, \oplus)$ is $R+J,(i) .\left(u_{-}, v_{-}\right) \in \mathrm{I}, 1-u_{+}+v_{+}=1$

a contact discontinuity $J$. We now consider the interaction between the shock $S$ and the rarefaction wave $R_{1}$. We can know that the shock $S$ is determined by

$$
\left\{\begin{array}{l}
\frac{x}{t}=\frac{1}{P^{2}}, \\
\frac{\mathrm{d} x}{\mathrm{~d} t}=\frac{1}{P P_{m^{\prime}}}, \\
\left.x\right|_{t=t_{2}}=x_{2},
\end{array}\right.
$$

where the point $A_{2}\left(x_{2}, t_{2}\right)$ can be calculated by above methods.

So, we have the following lemma

Lemma 4.4. The $\delta$-shock cannot penetrate the rarefaction wave $R_{2}$ and changes into a contact discontinuity $J$ and a shock $S$ at point $A_{2}$ after the interaction with $R_{2}$. The shock $S$, determined by (4.27), cannot penetrate the rarefaction wave $R_{1}$ and has $x=\frac{1}{P_{m^{\prime}}^{2}} t$ as its asymptote.

According to the Lemma 4.4, as $t \rightarrow \infty$, the solution can be described as

$$
\left(u_{-}, v_{-}\right)+R+\left(u_{m^{\prime}}, v_{m^{\prime}}\right)+J+\left(u_{+}, v_{+}\right) .
$$

(ii). $\left(u_{-}, v_{-}\right) \in \mathrm{I}, 1-u_{+}+v_{+}=1$, see Fig. 7 . In this kind, the $\delta$-shock interacts with the rarefaction wave $R_{2}$ at point $A(\bar{x}, \bar{t})$, which is calculated by (4.3), then changes into a new $\delta$-shock. Similar to Lemma 4.2 , we also have the following lemma. 

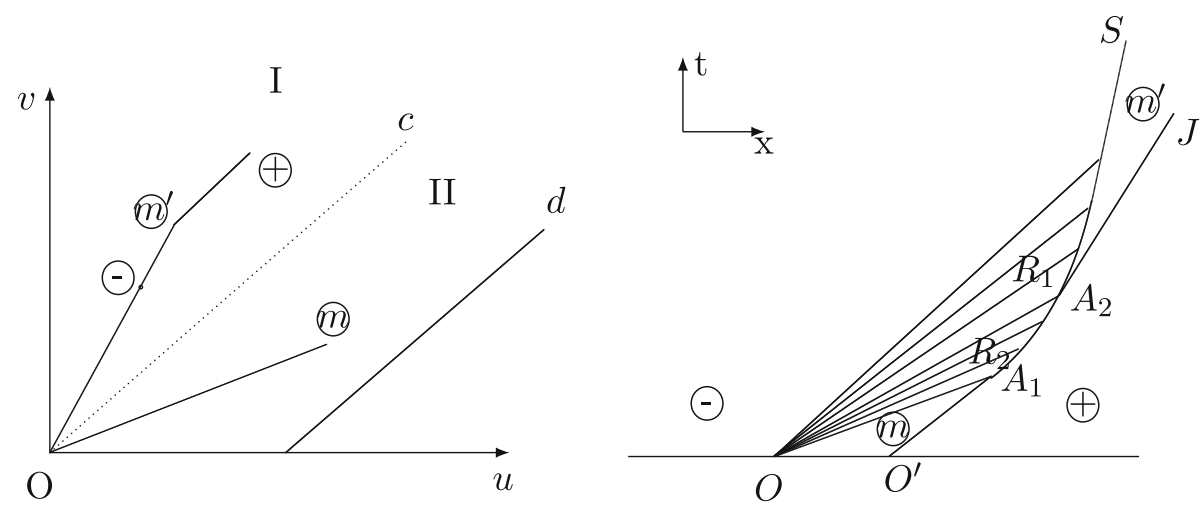

FIG. 8. Case 3.2: $\mathcal{R}(\odot, \oplus)$ is $S+J$

Lemma 4.5. The new $\delta$-shock cannot penetrate the rarefaction wave $R_{2}$ and has $x=t$ as its asymptote.

As $\varepsilon \rightarrow 0$, the solution can be described as

$$
\left(u_{-}, v_{-}\right)+R_{1}+J+\left(u_{+}, v_{+}\right) .
$$

We can see that the limit of the solution of (1.1) and (4.1) is the corresponding Riemann solution of (1.1) and (2.1).

Case 3.2. $\mathcal{R}(\ominus, \oplus)$ is $S+J$, see Fig. 8. Unlike subcase 3.1 (i), the $\delta$-shock penetrates the rarefaction wave $R_{2}$, then interacts with the $R_{1}$ and penetrates it. We will prove the following lemma.

Lemma 4.6. After penetrating the rarefaction wave $R_{2}$, the $\delta$-shock changes into a contact discontinuity $J$ and a shock $S$. Moreover the shock interacts with the rarefaction wave $R_{1}$ and penetrates it.

Proof. As the mentioned above, when the $\delta$-shock penetrates the $R_{2}$ at point $A_{2}\left(x_{2}, t_{2}\right)$, the $\delta$-shock entropy condition is not satisfied and its strength is zero, and we have a new Riemann problem, which is solved by a contact discontinuity $J$ and a shock $S$, where the shock $S$ is determined by $(4.27), \frac{1}{P_{-}} \leq \frac{1}{P} \leq 1$. The propagating speed of the shock is $\sigma=\frac{\mathrm{d} x}{\mathrm{~d} t}=\frac{1}{P P_{m^{\prime}}}$ and one can obtain $\frac{d^{2} x}{\mathrm{~d} t^{2}}<0$, which means the $\sigma$ decelerate in the penetration. We also have $\frac{1}{P_{-} P_{m^{\prime}}}<\frac{1}{P_{-}^{2}}$, which implies that the shock can penetrate the $R_{1}$.

When $t \rightarrow \infty$, it is easy to obtain that the solution can be described as

$$
\left(u_{-}, v_{-}\right)+S+\left(u_{m^{\prime}}, v_{m^{\prime}}\right)+J+\left(u_{+}, v_{+}\right) .
$$

which is the same as the corresponding Riemann solution.

Case 4 . $\delta$-shock $\oplus \delta$-shock.

In this case, we have $\left(u_{-}, v_{-}\right) \in \mathrm{II}, 1-u_{m}+v_{m}=1$ and $\left(u_{+}, v_{+}\right) \in \mathrm{I}$, see Fig. 9 . We can check that the propagating speed of the $\delta_{1}$-shock $\sigma_{1}=\frac{1}{P_{-} P_{m}}$ is greater than the speed of the $\delta_{2}$-shock $\sigma_{2}=\frac{1}{P_{m} P_{+}}$. The $\delta_{1}$-shock will overtake the $\delta_{2}$-shock at point $A(\bar{x}, \bar{t})$, which can be expressed as follows

$$
\left\{\begin{array}{l}
\frac{x}{t}=\frac{1}{P_{-} P_{m}}, \\
\frac{x-\varepsilon}{t}=\frac{1}{P_{m} P_{+}} .
\end{array}\right.
$$



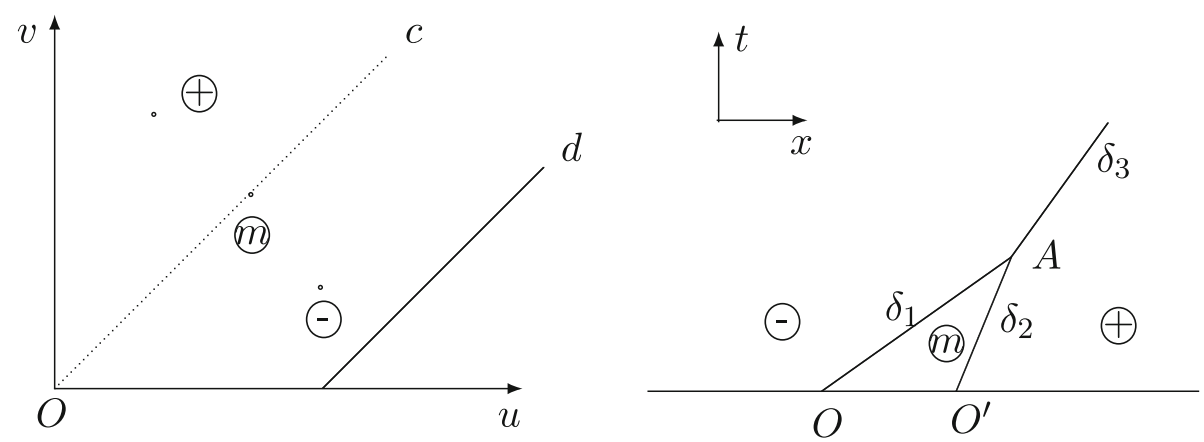

FIG. 9. Case 4: $\delta$-shock $\oplus \delta$-shock

So, we obtain

$$
(\bar{x}, \bar{t})=\left(\frac{P_{+}}{P_{+}-P_{-}} \varepsilon, \frac{P_{-} P_{m} P_{+}}{P_{+}-P_{-}} \varepsilon\right) .
$$

After the time $t=\bar{t}$, the $\delta$-shock entropy condition is satisfied

$$
\lambda_{2}^{+}=\frac{1}{P_{+}^{2}} \leq \lambda_{1}^{+}=\frac{1}{P_{+}} \leq \sigma_{3}=\frac{1}{P_{-} P_{+}} \leq \lambda_{1}^{-}=\frac{1}{P_{-}} \leq \lambda_{2}^{-}=\frac{1}{P_{-}^{2}},
$$

and a new $\delta$-shock is formed, denoted by $\delta_{3}$-shock, which is determined by

$$
\left\{\begin{array}{l}
\frac{\mathrm{d} x}{\mathrm{~d} t}=\frac{1}{P_{-} P_{+}} \\
\left.x\right|_{t=\bar{t}}=\bar{x}
\end{array}\right.
$$

Thus, as $t \rightarrow \infty$, the result of interaction of two $\delta$-shock waves is still a single $\delta$-shock wave. It is easy to see that as $\varepsilon \rightarrow 0$, the limit of the solution of (1.1) and (4.1) is the corresponding Riemann solution of (1.1) and (2.1).

Case 5. $R+J \oplus \delta$-shock. In this case, on the basis of the fact that $\mathcal{R}(\Theta$, (m) is $R+J$ and $\mathcal{R}(\mathrm{m}, \oplus)$ is a nonclassical $\delta$-shock, we have $1-u_{m}+v_{m}=1$ and $\left(u_{-}, v_{-}\right),\left(u_{+}, v_{+}\right) \in$ I. So, we divide this case into two subcases.

Case 5.1. $\mathcal{R}(\Theta, \oplus)$ is $R+J$. As discussed above, after the $\delta$-shock interacts with the right-contact discontinuity $J$ at the point $A$, we can see that $\frac{1}{P_{-}} \leq \frac{1}{P} \leq 1$ and the $\delta$-shock entropy condition is not satisfied and its strength is zero. Hence, we have a new Riemann problem and the $\delta$-shock changes into $S+J$. The shock begins interacting with the $R$ at the point $A$. We derive the equation of the shock $S: x=x(t)$ as

$$
\left\{\begin{array}{l}
\frac{x}{t}=\frac{1}{P^{2}}, \quad \frac{1}{P_{m^{\prime}}} \leq \frac{1}{P} \leq 1, \\
\frac{\mathrm{d} x}{\mathrm{~d} t}=\frac{1}{P P_{m^{\prime}}}, \\
x(\bar{t})=\bar{x} .
\end{array}\right.
$$

By virtue of the Lemma 4.4, we obtain that the shock $S$ cannot penetrate the rarefaction wave $R$ and has $x=\frac{1}{P_{m^{\prime}}^{2}} t$ as its asymptote (Fig. 10).

We can see that as $\varepsilon \rightarrow 0$, the solution can be described as

$$
\left(u_{-}, v_{-}\right)+R+\left(u_{m^{\prime}}, v_{m^{\prime}}\right)+J+\left(u_{+}, v_{+}\right) \text {. }
$$



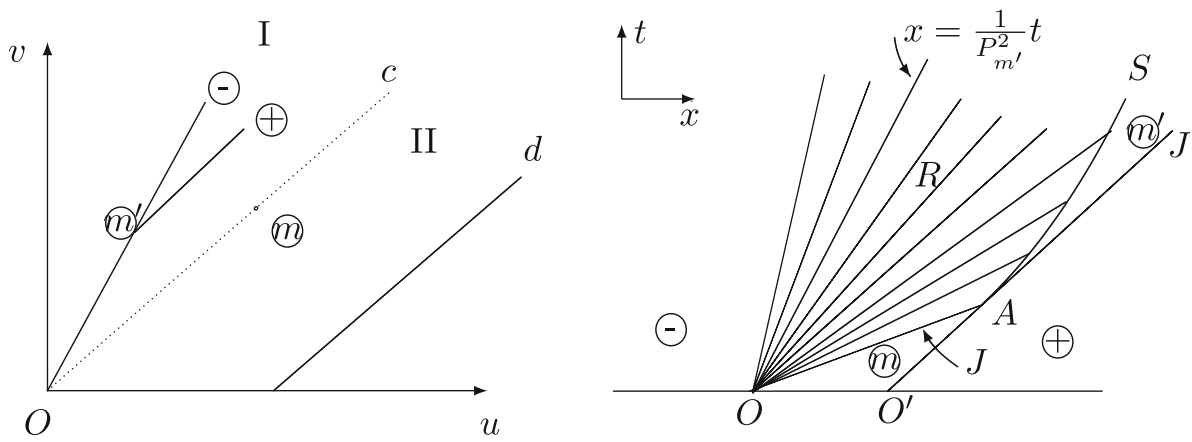

FIG. 10. Case 5.1: $\mathcal{R}(\odot, \oplus)$ is $R+J$
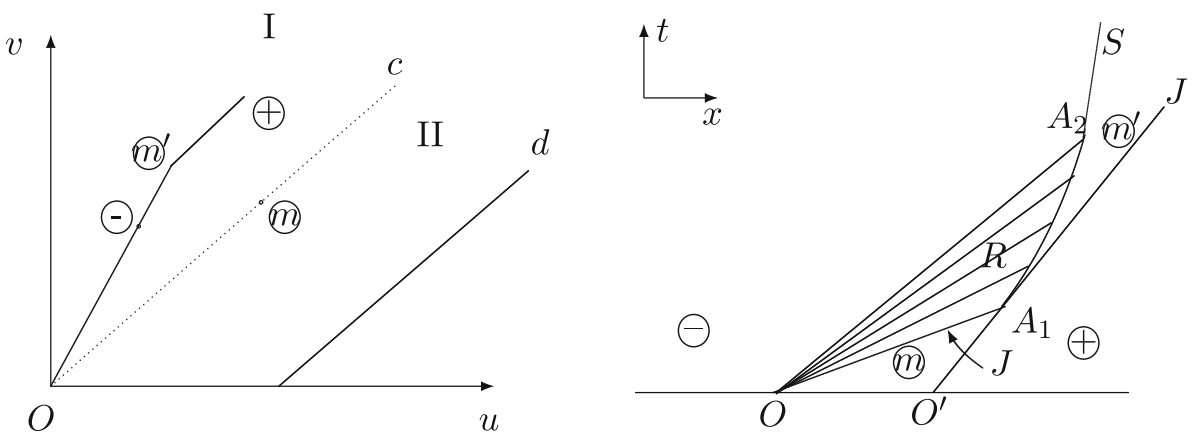

FIG. 11. Case 5.2: $\mathcal{R}(\odot, \oplus)$ is $S+J$

Case 5.2. $\mathcal{R}(\Theta, \oplus)$ is $S+J$. Similarly, we obtain that the $\delta$-shock interacts with the contact discontinuity at the point $A_{1}$, then changes into a shock $S$ and a new contact discontinuity. By Lemma 4.6, we obtain that the shock $S$ can penetrate the rarefaction wave $R$ at some point $A_{2}$, the solutions to this subcase are shown in Fig. 11.

We can see that as $t \rightarrow \infty$, the solution can be described as

$$
\left(u_{-}, v_{-}\right)+S+\left(u_{m^{\prime}}, v_{m^{\prime}}\right)+J+\left(u_{+}, v_{+}\right),
$$

which is the same as the corresponding Riemann solution.

So far, we have finished the discussion for the interactions of the $\delta$-shock and the elementary waves and the global solutions for the perturbed initial value problem (1.1) and (4.1) have been constructed. We summarize our results in the following.

Theorem 4.7. The limits of the perturbed Riemann solution of (1.1) and (4.1) are exactly the corresponding Riemann solution of (1.1) and (2.1). The Riemann solutions of (1.1) and (2.1) are stable and admissible with respect to such small perturbations of the initial data.

\section{Acknowledgments}

The author would like to thank Dr. Geng Lai for his helpful discussion and the anonymous referees who provided valuable comments and suggestions resulting in improvements in this paper. 
Open Access. This article is distributed under the terms of the Creative Commons Attribution License which permits any use, distribution, and reproduction in any medium, provided the original author(s) and the source are credited.

\section{References}

1. Ambrosio, L., Crippa, G., Figalli, A., Spinolo, L.A.: Some new well-posedness results for continuity and transport equations, and applications to the chromatography system. SIAM J. Math. Anal. 41, 1890-1920 (2009)

2. Ancona, F., Goatin, P.: Uniqueness and stability of $L^{\infty}$ solutions for Temple class systems with boundary and properties of the attenaible sets. SIAM J. Math. Anal. 34, 28-63 (2002)

3. Barti, P., Bressan, A.: The semigroup generated by a Temple class system with large data. Differ. Integr. Equ. 10, 401418 (1997)

4. Bianchini, S.: Stability of $L^{\infty}$ solutions for hyperbolic systems with coinciding shocks and rarefactions. SIAM J. Math. Anal. 33, 959-981 (2001)

5. Bouchut, F.: On zero pressure gas dynamics. In: Perthame, B. (ed.) Advances in Kinetic Theory and Computing. Series on Advances in Mathematics for Applied Sciences, vol. 22, World Scientific Publishing, River Edge, NJ, pp. 171-190 (1994)

6. Bressan, A., Goatin, P.: Stability of $L^{\infty}$ solutions of Temple class systems. Differ. Integr. Equ. 13, 1503-1528 (2000)

7. Chang, T., Hsiao, L.: The Riemann Problem and Interaction of Waves in Gas Dynamics, Pitman Monographs and Surveys in Pure and Applied Mathematics 41, Longman Scientific and Technical, Harlow (1989)

8. Chen, G.Q., Liu, H.: Formation of $\delta$-shock and vacuum states in the vanishing pressure limit of solutions to the Euler equations for isetropic fluids. SIAM J. Math. Anal. 34, 925-938 (2003)

9. Cheng, H.J., Yang, H.C.: Delta shock waves in chromatography equations. J. Math. Anal. Appl. 380, 475-485 (2011)

10. Dafermos, C.M.: Hyperbolic Conservation Laws in Continuum Physics, Grundlehren Math. Wiss.. Springer, Berlin (2000)

11. Dal Maso, G., Lefloch, P.G., Murat, F.: Definition and weak stability of nonconservative products. J. Math. Pures Appl. 74, 483-548 (1995)

12. Danilov, V.G., Shelkovich, V.M.: Dynamics of progation and interaction of $\delta$-shock waves in conservation law systems. J. Differ. Equ. 221, 333-381 (2005)

13. Danilov, V.G., Shelkovich, V.M.: Delta-shock wave type solution of hyperbolic systems of conservation laws. Q. Appl. Math. 63(3), 401-427 (2005)

14. Hayes, B.T., Lefloch, P.G.: Measure solutions to a strictly hyperbolic system of conservation laws. Nonlinearity 9, 15471563 (1996)

15. Huang, F., Wang, Z.: Well-posedness for pressureless flow. Commun. Math. Phys. 222, 117-146 (2001)

16. Kalisch, H., Mitrovic, D.: Singular solutions of a fully nonlinear $2 \times 2$ system of conservation laws. http://arXiv:1105.4640v3 (2011)

17. Keyfitz, B.L., Kranzer, H.C.: Spaces of weighted measures for conservation laws with singular shock solutions. J. Differ. Equ. 118, 420-451 (1995)

18. Korchinski, D.J.: Solution of a Riemann problem for a system of conservation laws possessing noclassical weak solution. Thesis, Adelphi University (1977)

19. Li, J.Q., Warnecke, G.: Generalized characteristics and the uniqueness of entropy solutions to zero-pressure gas dynamics. Adv. Differ. Equ. 8, 961-1004 (2003)

20. Li, J.Q., Yang, H.C.: Delta-shocks as limits of vanishing viscosity for multidimensional zero-pressure flow in gas dynamics. Q. Appl. Math. 59, 315-342 (2001)

21. Li, J.Q., Zhang, T., Yang, S.L.: The Two-dimensional Riemann Problem in Gas Dynamics. Pitman Monographs 98, Longman, Harlow (1998)

22. Mazzotti, M.: Occurrence of a delta-shock in non-linear chromatography. Proc. Appl. Math. Mech. 7. doi:10.1002/pamm. 200700912

23. Mazzotti, M.: Nonclassical composition fronts in nonlinear chromatography: delta-shock. Ind. Eng. Chem. Res. 48, 7733$7752(2009)$

24. Mazzotti, M., Tarafder, A., Cornel, J., Gritti, F., Guiochond, G.: Experimental evidence of a delta-shock in nonlinear chromatography. J. Chromatogr. A 1217, 2002-2012 (2010)

25. Nedeljkov, M.: Delta and singular delta locus for one dimensional systems of conservation laws. Math. Methods Appl. Sci. 27, 931-955 (2004)

26. Nedeljkov, M.: Singular shock waves in interactions. Q. Appl. Math. 66, 281-302 (2008)

27. Nedeljkov, M.: Shadow waves: entropies and interactions for delta and singular shocks. Arch. Ratio. Mech. Anal. 197(2), 489-537 (2010) 
28. Nedeljkov, M., Oberguggenberger, M.: Interactions of delta shock waves in a strictly hyperbolic system of conservation laws. J. Math. Anal. Appl. 344, 1143-1157 (2008)

29. Panov, E.Y., Shelkovich, V.M.: $\delta$ '-shock waves as a new type of solutions to system of conservation laws. J. Differ. Equ. 228, 49-86 (2006)

30. Serre, D.: Solutions à variations bornées pour certains systèmes hyperboliques de lois de conservation. J. Differ. Equ. 68(2), 137-168 (1987)

31. Serre, D.: Systems of Conservation Laws I/II. Cambridge University Press, Cambridge (1999)/(2000)

32. Shelkovich, V.M.: $\delta$ - and $\delta$ '-shock types of singular solutions to systems of conservation laws and the transport and concentration processes. Uspekhi Mat. Nauk 63:3(381), 73-146 (2008)

33. Shen, C.: Wave interactions and stability of the Riemann solutions for the chromatography equations. J. Math. Anal. Appl. 365, 609-618 (2010)

34. Shen, C., Sun, M.: Formation of delta shocks and vacuum states in the vanishing pressure limit of Riemann solutions to the perturbed Aw-Rascle model. J. Differ. Equ. 249, 3024-3051 (2010)

35. Sheng, W., Zhang, T.: The Riemann problem for the transportation equations in gas dynamics. Mem. Am. Math. Soc. 137, 654 (1999)

36. Smoller, J.: Shock Waves and Reaction-Diffusion Equations, 2nd edn.. Springer, New York (1994)

37. Sun, M.: Delta Shock waves for the chromatography equations as self-similar viscosity limits. Q. Appl. Math. 69, 425443 (2011)

38. Tan, D., Zhang, T., Zheng, Y.: Delta-shock waves as limits of vanishing viscosity for hyperbolic systems of conservation laws. J. Differ. Equ. 112, 1-32 (1994)

39. Temple, B.: Systems of conservation laws with invariant submanifolds. Trans. Am. Math. Soc. 280(2), 781-795 (1983)

40. Shelkovich, V.M.: The Riemann problem admitting $\delta$-, $\delta$ '-shocks and vacuum states (the vanishing viscosity approach). J. Differ. Equ. 231, 459-500 (2006)

41. Weinan, E., Rykov, Y.G., Sinai, Y.G.: Generalized variational principles, globalweak solutions and behavior with random initial data for systems of conservation laws arising in adhesion particle dynamics. Commun. Math. Phys. 177, 349380 (1996)

42. Yang, H.: Riemann problems for a class of coupled hyperbolic systems of conservation laws. J. Differ. Equ. 159, 447484 (1999)

43. Zeldovich, Y.B., Myshkis, A.D.: Elements of Mathematical Physics: Medium Consisting of Noninteracting Particles (in Russian). Nauka, Moscow (1973)

Guodong Wang

Department of Mathematics and Physics

Anhui University of Architecture

Hefei 230601

People's Republic of China

e-mail: yxgdwang@163.com

(Received: May 25, 2011; revised: December 14, 2012) 